PROCEEDINGS OF THE

AMERICAN MATHEMATICAL SOCIETY

Volume 132, Number 2, Pages 375-379

S 0002-9939(03)07184-3

Article electronically published on September 5, 2003

\title{
REDUCTION OF OPIAL-TYPE INEQUALITIES TO NORM INEQUALITIES
}

\author{
GORD SINNAMON
}

(Communicated by Jonathan M. Borwein)

\begin{abstract}
Weighted Opial-type inequalities are shown to be equivalent to weighted norm inequalities for sublinear operators and for nearly positive operators. Examples involving the Hardy-Littlewood maximal function and the nonincreasing rearrangement are presented.
\end{abstract}

Opial-type inequalities are related to norm inequalities much as quadratic forms are related to bilinear forms. A linear operator $T$ on Hilbert space gives rise to the bilinear form $(f, g) \mapsto\langle T f, g\rangle$ and the quadratic form $f \mapsto\langle T f, f\rangle$. Duality shows that the norm of $T$ and the norm of the bilinear form coincide, and a standard polarization argument shows that this norm is equivalent to but not necessarily equal to the norm of the quadratic form, called the numerical radius of $T$.

In this paper, far from the luxuries of Hilbert spaces and linear operators, we show that the equivalence of operator norm and numerical radius persists. The work is in response to Richard Brown's suggestion that Steven Bloom's result 2, Theorem 1] which gives the equivalence for positive operators should apply in greater generality. Opial-type inequalities have been much studied since Opial's original paper in 1960 and the papers [2, 3] and [4] include many references.

After the main theorem showing equivalence of Opial-type and norm inequalities, an example involving the Hardy-Littlewood maximal function is included to illustrate that the equivalence cannot be taken in a pointwise sense.

To show that the method can be readily applied to generate nontrivial inequalities from known norm inequalities we give a simple weight characterization of an Opial-type inequality for the nonincreasing rearrangement.

Begin with a $\sigma$-finite measure space and consider the real or complex-valued measurable functions. The simple functions are those that take finitely many values and vanish off a set of finite measure. A map $T$ taking simple functions to measurable functions is nearly positive provided there exists a $D>0$ such that

$$
|T f| \leq D(T g) \text { whenever }|f| \leq g
$$

and is sublinear provided there exists a $D>0$ such that

$$
|T(f+g)| \leq D(|T f|+|T g|)
$$

for all simple $f, g$.

Received by the editors July 19, 2002.

2000 Mathematics Subject Classification. Primary 26D15.

Key words and phrases. Opial inequality, Opial-type inequality, weight.

Supported by the Natural Sciences and Engineering Research Council of Canada. 
We write $\|f\|_{p}=\left(\int|f|^{p}\right)^{1 / p}$ for $0<p<\infty$ and $\|f\|_{\infty}=\operatorname{ess} \sup |f|$. Note that for all $f$ and $g,\|f+g\|_{p} \leq c(p)\left(\|f\|_{p}+\|g\|_{p}\right)$ where $c(p) \equiv \max \left(1,2^{(1 / p)-1}\right)$. Where it arises we take the product $0 \cdot \infty$ to be zero.

Theorem 1. Let $p, q$ and $r$ be positive real numbers and $u \geq 0$ and $0<v<\infty$ be measurable functions. Suppose that $T$ is nearly positive or sublinear. Then there exists a finite $C$ such that the weighted Opial-type inequality

$$
\int|T f|^{q}|f|^{r} u \leq C\|f v\|_{p}^{q+r}
$$

holds for all simple $f$ if and only if there exists a finite $B$ such that

$$
\int|T f|^{q}|g|^{r} u \leq B\|f v\|_{p}^{q}\|g v\|_{p}^{r}
$$

holds for all simple $f, g$. If $r \leq p$, then (2) holds if and only if the weighted norm inequality

$$
\left\|(T f) u^{1 / q} v^{-r / q}\right\|_{p q /(p-r)} \leq B^{1 / q}\|f v\|_{p}
$$

holds for all simple $f$.

Proof. It is clear that if (2) holds, then (11) holds. To prove the converse we suppose that (1) holds and fix simple functions $f$ and $g$. If $\|g v\|_{p}=0$, then $g=0$ almost everywhere; so (2) holds because both sides vanish. If $\|f v\|_{p}=0$, then $f=0$ almost everywhere. Since the underlying measure is $\sigma$-finite and $v<\infty$, there is a sequence of sets $E_{n} \subset\{x: v(x)<n\}$, each of finite measure, whose union is the whole space. If $T$ is nearly positive, then for any $\lambda>0$ we have

$$
\int|T(0)|^{q}\left|\lambda \chi_{E_{n}}\right|^{r} u \leq D^{q} \int\left|T\left(\lambda \chi_{E_{n}}\right)\right|^{q}\left|\lambda \chi_{E_{n}}\right|^{r} u \leq C D^{q}\left\|\lambda \chi_{E_{n}} v\right\|_{p}^{q+r}<\infty .
$$

Dividing by $\lambda^{r}$ and then letting $\lambda \rightarrow 0$ shows that $u|T(0)|^{q}$ is zero almost everywhere on each $E_{n}$ and hence $u T(0)$ vanishes almost everywhere. If $T$ is sublinear we have

$$
\begin{aligned}
\int|T(0)|{ }^{q}\left|\lambda \chi_{E_{n}}\right|^{r} u \leq & D^{q} 2^{q-1} \int\left|T\left(\lambda \chi_{E_{n}}\right)\right|^{q}\left|\lambda \chi_{E_{n}}\right|^{r} u \\
& +D^{q} 2^{q-1} \int\left|T\left(-\lambda \chi_{E_{n}}\right)\right|^{q}\left|-\lambda \chi_{E_{n}}\right|^{r} u \\
\leq & C D^{q} 2^{q}\left\|\lambda \chi_{E_{n}} v\right\|_{p}^{q+r}<\infty .
\end{aligned}
$$

Again we conclude that $u T(0)$ vanishes almost everywhere. Therefore, whether $T$ is nearly positive or sublinear, we see that (2) holds when $\|f v\|_{p}=0$ because both sides vanish.

In the remaining case both $\|f v\|_{p}$ and $\|g v\|_{p}$ are positive and we may assume that both are finite since otherwise (2) holds with infinite right-hand side. The inequality (2) is $r$-homogeneous in $g$; so we may normalize $g$ so that $\|g v\|_{p}=\|f v\|_{p}$. If $T$ is nearly positive, set $h=|f|+|g|$ and note that $|f| \leq h,|g| \leq h$, and $\|h v\|_{p} \leq 2 c(p)\|f v\|_{p}$. We have

$$
\int|T f|^{q}|g|^{r} u \leq D^{q} \int|T h|^{q}|h|^{r} u \leq C D^{q}\|h v\|_{p}^{q+r} \leq(2 c(p))^{q+r} C D^{q}\|f v\|_{p}^{q}\|g v\|_{p}^{r},
$$


which gives (2). If $T$ is sublinear, then set

$$
\begin{aligned}
& h_{1}= \begin{cases}2 f+3|g|, & \operatorname{Re}(f)+|g| \geq 0, \\
3 f+|g|, & \operatorname{Re}(f)+|g|<0,\end{cases} \\
& h_{2}= \begin{cases}-f-3|g|, & \operatorname{Re}(f)+|g| \geq 0, \\
-2 f-|g|, & \operatorname{Re}(f)+|g|<0\end{cases}
\end{aligned}
$$

and check that $f=h_{1}+h_{2},|g| \leq\left|h_{1}\right|,|g| \leq\left|h_{2}\right|,\left\|h_{1} v\right\|_{p} \leq 6 c(p)\|f v\|_{p}$, and $\left\|h_{2} v\right\|_{p} \leq 6 c(p)\|f v\|_{p}$. We have

$$
\begin{aligned}
\int|T f|^{q}|g|^{r} u & \leq D^{q} 2^{q-1} \int\left|T\left(h_{1}\right)\right|^{q}\left|h_{1}\right|^{r} u+D^{q} 2^{q-1} \int\left|T\left(h_{2}\right)\right|^{q}\left|h_{2}\right|^{r} u \\
& \leq C D^{q} 2^{q-1}\left\|h_{1} v\right\|^{q+r}+C D^{q} 2^{q-1}\left\|h_{2} v\right\|^{q+r} \\
& \leq C D^{q} 2^{q}(6 c(p))^{q+r}\|f v\|_{p}^{q}\|g v\|_{p}^{r} .
\end{aligned}
$$

This completes the proof of the equivalence of (1) and (2).

To see that (2) implies (3) in the case $r \leq p$ take the supremum of both sides of (2) over all simple $g$ with $\|g v\|_{p} \leq 1$ and then take $q$ th roots. The converse follows by Hölder's inequality with indices $p /(p-r)$ and $p / r$. This completes the proof.

The same proof shows the equivalence of (11) and (2) when the norm $\|\cdot\|_{p}$ is replaced by any Banach Function Space norm.

There is no simple analogue of (3) in the case $r>p$ because if the underlying measure is non-atomic, then (2) can hold only if $u T$ is trivial, but for atomic measures, nontrivial inequalities of the form (2) may hold when $r>p$.

Suppose now that $r \leq p$. The implication (3) $\Longrightarrow(1)$ holds pointwise. That is, if (3) holds for a particular function or class of functions, then (1) holds for that function or that class. It is important to point out that the implication (11) $\Longrightarrow$ (3) does not hold pointwise. To illustrate this point we fix a positive weight $w$, take $p>1, q=1, r=p-1, u=w, v=w^{1 / p}$ and let $T=M$, the Hardy-Littlewood maximal function on $\mathbf{R}$. This operator, defined by

$$
M f(x)=\sup _{x \in(a, b)} \frac{1}{b-a} \int_{a}^{b}|f|,
$$

is clearly positive. The norm inequality corresponding to (3) is

$$
\int_{-\infty}^{\infty}(M f)^{p} w \leq B^{p} \int_{-\infty}^{\infty}|f|^{p} w
$$

and the Opial-type inequality corresponding to (11) is

$$
\int_{-\infty}^{\infty}(M f)|f|^{p-1} w \leq C \int_{-\infty}^{\infty}|f|^{p} w .
$$

If we restrict our attention to the class of functions $\left\{w^{1-p^{\prime}} \chi_{(a, b)}: a<b\right\}$, then (4) implies

$$
\int_{a}^{b} M\left(w^{1-p^{\prime}} \chi_{(a, b)}\right)^{p} w \leq B^{p} \int_{a}^{b} w^{1-p^{\prime}}
$$

and (5) becomes

$$
\int_{a}^{b} M\left(w^{1-p^{\prime}} \chi_{(a, b)}\right) \leq C \int_{a}^{b} w^{1-p^{\prime}}
$$


Considered as conditions on the weight $w$, the theorem above shows that (4) and (5) are equivalent. Using [6, IX.4.1 and IX.8.13] we see that (44), (5) and (6) are all equivalent to Muckenhoupt's $A_{p}$ condition. The weight condition (17), however, is genuinely weaker than $A_{p}$. Specifically, the weight $w(x)=|x|^{-1}$ is not in $A_{p}$ (see [6, IX.4.4]), but we argue that it does satisfy (7). By symmetry it is enough to prove (7) for intervals $(a, b)$ satisfying $-b \leq a<b$ and in this case we have

$$
\begin{aligned}
\int_{a}^{b} M\left(|x|^{p^{\prime}-1} \chi_{(a, b)}\right) d x & \leq \int_{a}^{b} b^{p^{\prime}-1} d x \\
& \leq 4 \int_{\max (b / 2, a)}^{b} b^{p^{\prime}-1} d x \\
& \leq 4 \int_{\max (b / 2, a)}^{b}(2 x)^{p^{\prime}-1} d x \\
& \leq 2^{p^{\prime}+1} \int_{a}^{b}|x|^{p^{\prime}-1} d x
\end{aligned}
$$

Thus, the requirement that the Opial-type inequality (5) hold for this class of functions is a strictly weaker condition on $w$ than requiring that the norm condition (44) hold for this class. We thank the referee for suggesting this example.

For $f \geq 0$ defined on $[0,1]$, let $f^{*}$ denote the nonincreasing rearrangement of $f$. The operator $f \mapsto f^{*}$ is positive. So we can use the above theorem to understand the relationship between $f$ and $f^{*}$.

Example 2. If $u \geq 0$ and $v>0$, then there exists $a>0$ such that

$$
\int_{0}^{1} f^{*} f u \leq C \int_{0}^{1} f^{2} v
$$

holds for all $f \geq 0$ if and only if

$$
\left(\int_{0}^{x} u^{2} / v\right)\left(\int_{1-x}^{1} v^{*}\right)^{-1}
$$

is a bounded function of $x \in[0,1]$.

Proof. Take $T f=f^{*}, p=2, q=r=1$ and replace $v$ by $v^{1 / 2}$ in the theorem to see that (8) holds if and only if there is a $B$ such that

$$
\int_{0}^{1}\left(f^{*}\right)^{2} u^{2} / v \leq B^{2} \int_{0}^{1} f^{2} v
$$

holds for all $f \geq 0$. (The passage from simple functions to all functions is standard.) Since the left-hand side of (10) is unaffected when $f$ is replaced by something equimeasurable, we may replace the right-hand side by

$$
B^{2} \inf \left\{\int_{0}^{1} g^{2} v: g \text { equimeasurable with } f\right\} .
$$

An application of [1. Theorem II.2.2] shows that this is

$$
B^{2} \int_{0}^{1}\left(f^{*}\right)^{2} v^{\star}
$$


where $v^{\star}$ denotes the nondecreasing rearrangement of $v$. With this observation, (10) reduces to the inequality

$$
\int_{0}^{1} F u^{2} / v \leq B^{2} \int_{0}^{1} F v^{\star}
$$

for all nonincreasing $F$, and it is easy to deduce (or see [5, Proposition 1]) that this is equivalent to the boundedness of

$$
\left(\int_{0}^{x} u^{2} / v\right)\left(\int_{0}^{x} v^{\star}\right)^{-1}
$$

for $x \in[0,1]$. Since $v^{\star}(x)=v^{*}(1-x)$, this is (9)).

This example readily extends to general indices $p, q$ and $r<p$ by the same method.

\section{REFERENCES}

1. C. Bennett and R. Sharpley, Interpolation of Operators, Pure and Applied Mathematics, Vol. 129, Academic Press, Boston, MA, 1988. MR 89e:46001

2. S. Bloom, First and second order Opial inequalities, Studia Math. 126 (1997), 27-50. MR 98g:26016

3. R. C. Brown, A. M. Fink, and D. B. Hinton, Some Opial, Lyapunov, and De la Valée Poussin inequalities with nonhomogeneous boundary conditions, J. Inequal. Appl. 5 (2000), 11-37. MR 2000m:34073

4. G. Sinnamon, Weighted Hardy and Opial-type inequalities, J. Math. Anal. Appl. 160 (1991), 434-445. MR 92f:26037

5. V. D. Stepanov, The weighted Hardy's inequality for nonincreasing functions, Trans. Amer. Math. Soc., 338 (1993), 173-186. MR 93j:26012

6. A. Torchinsky, Real-Variable Methods in Harmonic Analysis, Academic Press, Orlando, FL, 1986. MR 91m:42001

Department of Mathematics, University of Western Ontario, London, Ontario, N6A 5B7, CANADA

E-mail address: sinnamon@uwo.ca 